\title{
Measures of Location Effectiveness of Vibration Absorbers
}

\author{
Jerome K. Vethecan \\ BAE Systems Australia, 677 Victoria Street, Abbotsford, Victoria, 3067, Australia
}

\begin{abstract}
Aleksandar Subic
Department of Mechanical E Manufacturing Engineering, Royal Melbourne Institute of Technology, PO Box 71, Bundoora, Victoria, 3083, Australia
\end{abstract}

(Received 19 December 2001; accepted 21 March 2001)

\begin{abstract}
The use of dynamic vibration absorbers is common in engineering applications where there is a need to attenuate high vibration levels due to resonant response. Criteria for the optimal specification of absorber properties are well known, being specified in terms of natural frequency, mass ratio and damping. Apart from this, the selection of the most effective attachment location is an important function of the design, and will be subject to the structural dynamics of the system to be treated. While it is commonly held that anti-nodal locations are best for attachment, the mode shape does not provide a true measure of the effectiveness when the device is attached elsewhere. In this paper, the issue of determining the location-effectiveness of dynamic vibration absorbers is examined using structural dynamic coupling principles. Specifically, the modal-coupling method which utilises modal models of both primary and auxiliary systems is considered. A measure of effectiveness is developed using synthesised frequency response functions of the coupled system to indicate the effectiveness of the device over a subset of response coordinates and candidate attachment locations. This information supplements the mode shape and in addition provides a tool for the analyst to make a quantitative assessment of all available options, which is especially useful when the attachment options are severely constrained.
\end{abstract}

\section{NOMENCLATURE}

$[M],[C],[K]$ - mass, damping and stiffness matrices of a subsystem

$\left[C_{g}\right],\left[K_{g}\right]$ - global coupling damping and stiffness matrices connecting subsystems

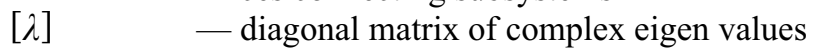

$[\psi] \quad$ - eigen vector matrix with arbitrary scaling

$[\varphi] \quad-$ mass-normalised eigen vector matrix

$\psi_{i}, \varphi_{i} \quad-i$-th eigen vector from matrices $[\psi]$ and $[\varphi]$ respectively

$x_{j} \quad-$ response at $j$-th degree of freedom in physical coordinate system

$u_{i} \quad$ - response at $i$-th degree of freedom in modal coordinate system

$f_{k} \quad$ - harmonic excitation at $k$-th degree of freedom in physical coordinate system

$\omega \quad$ - frequency, $\mathrm{rad} / \mathrm{s}$

$h_{j k}(\omega)$ - complex frequency response function, response at $j$-th degree of freedom to harmonic excitation at $k$-th degree of freedom

$\mathbf{0}$

- block matrix of zeros, of compatible dimension

\section{INTRODUCTION}

Dynamic vibration absorbers are commonly used in machines and structures for attenuating high-amplitude vibration responses due to resonant conditions. These devices are effectively an auxiliary dynamic system attached to the primary system. When the auxiliary system is 'tuned' to the resonant mode of the primary system, this influences the dynamic characteristics of the primary system by introducing an additional natural frequency and an anti-resonance. The idea is to alter the characteristics of the primary system so that attenuation is achieved over the frequency range in which the excitation occurs. Dynamic absorbers are specified by a welldefined set of system properties, which include mass, frequency tuning and damping. If these parameters are fixed, in the sense that they are invariant during normal operation, then the dynamic absorber is said to be passive. Successful applications of passive devices are plentiful, and can be found in automotive and aircraft structures, civil engineering structures such as tall towers and bridges, machines and machine foundations, ship structures, power transmission cables, heavy machinery and elsewhere. ${ }^{1,7-9,12}$

The effective bandwidth of attenuation on the primary structure depends on the dynamic properties of the auxiliary system. Optimum tuning of the dynamic parameters lead to results which are well known, and is driven by the requirements of the application., ${ }^{9,11,12}$ The type of tuning is contingent on whether the excitation occurs at a constant frequency or whether it sweeps across a wider band. In general, although there may be exceptions, the former case benefits from a lightly-damped tuning, while the latter case depends on a more optimally selected damping. Rules about the selection of appropriate optimal mass and stiffness are commonly found in standard engineering literature.

Quite apart from optimal dynamical properties, the question of where physically to attach the dynamic absorber to the primary system is very important if one is to maximise its attenuation performance. Experiential evidence strongly suggests that any anti-nodal locations of the mode(s) in question are good candidates for placing the dynamic absorber, and applications in references ${ }^{7-9}$ do support this. Assuming that an 